\title{
A Comparative Study of Arch Width, Overjet and Overbite between Bilateral Congenital Missing Lateral Incisor and Normal Class I Occlusion
}

\author{
Zena Hekmat Altaee ${ }^{1}$, Laith Hamood Aswad AL-Salmany ${ }^{2}$, Maha Mishaal Turki ${ }^{3}$ \\ ${ }^{1}$ Ass. Prof., ${ }^{2}$ Assist. Lect., Department of Orthodontics, College of Dentistry, University of Anbar, Iraq, \\ ${ }^{3}$ Assist., Department of Prosthodontics, College of Dentistry, University of Anbar, Iraq
}

\begin{abstract}
Permanent lateral incisors have been considered as major missing teeth in the mouth. The main aim of the present study is to conduct a comparative study of maxillary and mandibular arch widths, overjet and overbite for a sample with missing lateral incisors and normal class I occlusion.

The sample of the study comprises sixty pairs of study models of both genders, which were separated into two groups. The first group consisted of thirty casts of bilateral congenital missing lateral incisors, while the second group consisted of thirty casts of the class I occlusion. The intercanine width, interpremolar width, intermolar width, overjet and overbite were measured for the sixty samples.

The results of this study demonstrate a significant reduction in maxillary and mandibular arch width in bilateral missing lateral Incisors as compared with normal class I group. The results also revealed a significant reduction in overjet and overbite in bilateral missing lateral incisors as compared with normal class I group. A significant difference was also reported between the two groups based on gender difference in the maxillary arch width. Conversely, non-significant difference was found in the mandibular arch width. The present study also shows non-significant difference based on gender difference in overjet and overbite in the two groups.
\end{abstract}

Keywords: Congenital missing teeth, lateral incisors, Arch width, class I.

\section{Introduction}

The missing lateral incisors has been considered as major congenital dental anomaly ${ }^{(1,2,3)}$. Missing teeth results in disturbances in developing occlusions, masticator verbal dysfunctions, and it affects aesthetics as well. Thus, a considerable number of associated consultants is required to cure such cases ${ }^{(4)}$. However, missing teeth etiology remains indefinite and requires more research. Nevertheless, it seems that a congenital missing tooth is thought to be strongly controlled by genes, and is associated with various syndromes ${ }^{(4)}$. It is commonly believed that upper lateral side incisors (excluding the third molar) are the most reported cases of missing teeth with incidence $5 \%{ }^{(5-6)}$.

Furthermore, the orthodontic treatments of patients experiencing bi-or unilateral congenitally missing lateral incisors has been considered as one of the challenges affecting the treatment plan. The two main alternates, the orthodontic close the space or open the space regarding the prosthetic replacements, might be comprising functions, periodontal health, as well as aesthetics ${ }^{(7)}$.

Arch width discrepancy is an important diagnostic aid, which can expect the treatment outcomes of an orthodontic cases ${ }^{(8)}$. It is important for orthodontist to have information of the normal growth, dentition development, arch dimensions, and any variations from the normal ${ }^{(9)}$.

The present study focuses on the study of maxillary and mandibular arch width, overjet and overbite of patients with bilateral missing lateral incisors and normal class I occlusion in both genders to identify differences in these two types of groups. 


\section{Materials and Method}

In the present study, sixty patients we chosen either from a private orthodontic clinic, or from Orthodontic Department, Faculty of Dentistry, University of Anbar. Patients must be between (15-35) years of age, and their age mean was $26.3 \pm 0.3$ years.

Care was taken to exclude the patients who have previous orthodontic treatment history, cleft lip and palate, serial extraction as well as history regarding the extraction of permanent teeth.

All the radiographs have been estimated on the dental viewer through one author to the patient. Furthermore, a tooth has been considered as congenitally missing lateral incisors in case no trace on radiograph is found. Treatment records were also checked to verify if the missing tooth was extracted or not. Impressions were taken by Alginate impression material and were poured by orthodontic plaster. All information about the patients ex. name, age, gender and radiograph were collected and attached to the records made for all patients.

Then the study sample was divided into two groups. The first group consisted of thirty pairs of patients with bilateral congenital missing lateral incisors equally divided between the genders (fifteen males and fifteen females), and the second group consisted of thirty pairs of patients with class I occlusion based on a class I skeletal relationship without any abnormality to be the control group of this study. These were also divided equally between the genders (fifteen females and fifteen males).

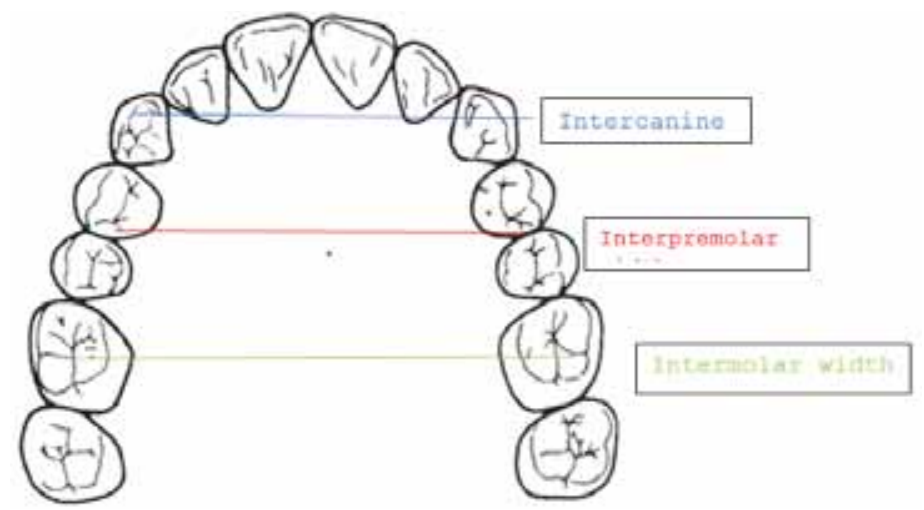

Figure 1: The landmarks used in this study

Statistical Analysis: The data were collected then statistically analyzed using SPSS software version 25 to calculate means and standard deviations of quantitatively
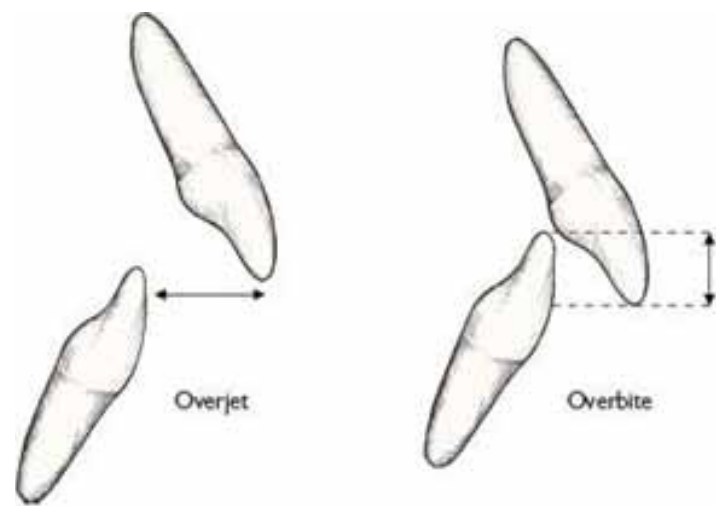

Measuring Technique: Measurements were made on the upper and lower study model by a single examiner using ecteronic digital caliper with sharpened tips which records up to $0.01 \mathrm{~mm}$. The dental arch width was recorded by measuring intercanine width, interpremolar width, intermolar width, overjet and overbite.

The reference points for the measurements were marked by using the sharp-pointed pencil to create the exact landmark points. The reference points that were used as landmarks are presented as follows and as shown in (figure 1):

1. Intercanine width: The distance between the cusp tips of the maxillary right permanent canines and left permanent canines.

2. Interpremolar width: The distance between the first premolar of the left side to the right side at the distal end of its occlusal groove.

3. Intermolar width: The distance between the maxillary first permanent molars of the left side to the same of the right side at the central fossae on the occlusal surface.

4. Over jet: The extent of horizontal overlap of the maxillary central incisor over the mandibular central incisor.

5. Over bite: The extent of vertical overlap of the maxillary central incisors over the mandibular central incisors.

collected data. The difference between the two means was identified using ( $\mathrm{t}$ ) test. The significance among means was calculated at $\mathrm{P}<0.05$ critical level. 
Reliability Test: The distance of twenty pairs of study models were measured randomly. After three weeks, the measurements were repeat to determine the reliability of the measurement by using intraclass correlation coefficient test, which showed excellent reliability $(>0.90)$.

\section{Results}

The chronological age range of the sample was (15$35)$ years. Table (1) displayed the results including the comparison of arch widths between bilateral congenital missing lateral incisors (BCMLI) with Class I normal occlusion in maxillary arch. The results indicated a significant difference between maxillary arch widths in both groups $(\mathrm{p}<0.05)$.

The comparison of arch widths between bilateral congenital missing lateral incisors (BCMLA) with Class I normal occlusion in mandibular arch is shown in the Table (2).

There was a significant difference in mandibular arch widths in both groups $(\mathrm{p}<0.05)$; however, intermolar distance shows non-significant difference $(p>0.05)$.

As shown in Table (3), there is highly significant reduction in overjet and overbite $(\leq 0.001)$ in the bilateral congenital missing lateral incisors (BCMLA) when compare with normal class I occlusion.

Based on the results tabulated in (4), there was a significant variance between male and female in two groups $(p<0.05)$ in terms of the intercanine width, interpremolar width and intermolar width in the maxillary arch.

Conversely, non-significant difference $p>0.05$ in terms of intercanine width, interpremolar width and intermolar width in mandibular arch was revealed.

Table (4) demonstrates non-significant differences ( $p>0.05$ ) according to gender in overjet and the overbite in the two groups.
Table (1) Illustrates the results of comparing arch widths between bilateral congenital missing lateral incisors (CMLA) and Class I normal occlusion in maxillary arch

\begin{tabular}{|l|l|c|c|c|}
\hline Variable & & BCMLA & Class I & P. value \\
\hline \multirow{2}{*}{ Intercanine Width } & Mean & 26.32 & 30.11 & \multirow{2}{*}{$0.001^{* *}$} \\
\cline { 2 - 5 } & S.D. & 0.8 & 1.32 & \\
\hline \multirow{2}{*}{ Interpremolar Width } & Mean & 33.7 & 36.5 & \multirow{2}{*}{$0.045^{*}$} \\
\cline { 2 - 5 } & S.D. & 1.6 & 1.32 & \\
\hline \multirow{2}{*}{ Intermolar Width } & Mean & 42.47 & 47.81 & \multirow{2}{*}{$0.037^{*}$} \\
\cline { 2 - 4 } & S.D. & 1.8 & 1.26 & \\
\hline
\end{tabular}

*Significant at $\mathrm{P}<0.05$., **Highly significant at $\mathrm{P} \leq 0.001$

Table (2) Shows the comparison of arch widths between bilateral congenital missing lateral incisors (CMLA) with Class I normal occlusion in mandibular arch.

\begin{tabular}{|l|l|c|c|c|}
\hline Variable & & BCMLA & Class I & P. value \\
\hline \multirow{2}{*}{ Intercanine Width } & Mean & 23.14 & 27.89 & \multirow{2}{*}{$0.035^{*}$} \\
\cline { 2 - 4 } & S.D & 1.32 & 0.71 & \\
\hline \multirow{3}{*}{ Interpremolar Width } & Mean & 31.6 & 32.4 & \multirow{2}{*}{$0.028^{*}$} \\
\cline { 2 - 4 } & S.D & 0.78 & 0.91 & \\
\hline \multirow{2}{*}{ Intermolar Width } & Mean & 40.87 & 43.2 & \multirow{2}{*}{0.069} \\
\cline { 2 - 4 } & S.D & 1.24 & 0.79 & \\
\hline
\end{tabular}

*Significant at $\mathrm{P}<0.05$.

Table (3) Shows the comparison of overjet and overbite between bilateral congenital missing lateral incisors (BCMLA) with Class I normal occlusion.

\begin{tabular}{|l|l|c|c|c|}
\hline Variable & & BCMLA & Class I & \multirow{2}{*}{ P. value } \\
\hline \multirow{2}{*}{ Overjet } & Mean & 1.98 & 3.1 & \multirow{2}{*}{$0.001^{*}$} \\
\cline { 2 - 4 } & S.D. & 0.81 & 0.68 & \\
\hline \multirow{2}{*}{ Overbite } & Mean & 0.89 & 2.11 & \multirow{2}{*}{$0.001^{*}$} \\
\cline { 2 - 4 } & S.D. & 0.56 & 0.81 & \\
\hline
\end{tabular}

$*$ Highly Significant at $\leq 0.001$.

Table (4) Reports gender effect results on bilateral congenital missing lateral incisors (BCMLA) with Class I normal occlusion.

\begin{tabular}{|l|c|c|c|c|c|c|}
\hline \multirow{2}{*}{ Variable } & \multicolumn{2}{|c|}{ BCMLA } & \multirow{2}{*}{ P. value } & \multicolumn{2}{|c|}{ Class I } \\
\cline { 2 - 3 } & Female Mean SD & Male Mean SD & & Female Mean SD & Male Mean SD \\
\hline Max. Intercanine & 28.54 & 30.12 & \multirow{2}{*}{$0.034^{*}$} & 31.76 & 32.8 \\
\cline { 2 - 3 } \cline { 5 - 6 } & 1.98 & 0.67 & & 1.92 & 2.12 \\
\hline
\end{tabular}




\begin{tabular}{|c|c|c|c|c|c|c|}
\hline Max. Interpremolar & 34.14 & 36.7 & \multirow{2}{*}{$0.00 * *$} & 35.8 & 38.1 & \multirow{2}{*}{$0.01 *$} \\
\hline Width & 1.87 & 1.67 & & 1.97 & 2.65 & \\
\hline Max. Intermolar & 41.1 & 43.1 & \multirow{2}{*}{$0.04 *$} & 44.5 & 48.12 & \multirow{2}{*}{$0.001 * *$} \\
\hline Width & 0.87 & 1.54 & & 1.78 & 2.34 & \\
\hline Man.Intercanine & 22.46 & 24.34 & \multirow{2}{*}{0.082} & 26.5 & 28.45 & \multirow{2}{*}{0.091} \\
\hline Width & 0.32 & 0.76 & & 1.32 & 0.65 & \\
\hline Man.Interpremolar & 30.8 & 31.78 & \multirow{2}{*}{0.067} & 32.1 & 32.98 & \multirow{2}{*}{0.075} \\
\hline Width & 0.87 & 0.43 & & 0.67 & 1.34 & \\
\hline Man.Intermolar & 40.1 & 41.12 & \multirow{2}{*}{0.092} & 43.23 & 44.1 & \multirow{2}{*}{0.086} \\
\hline Width & 1.29 & 1.98 & & 0.89 & 1.51 & \\
\hline Overjet & 1.67 & 2.1 & \multirow{2}{*}{0.072} & 3.65 & 3.53 & \multirow{2}{*}{0.065} \\
\hline Width & 0.67 & 0.32 & & 0.75 & 1.02 & \\
\hline Overbite & 1.94 & 0.86 & \multirow{2}{*}{0.065} & 3.12 & 2.96 & \multirow{2}{*}{0.078} \\
\hline Width & 0.78 & 1.25 & & 1.34 & 1.82 & \\
\hline
\end{tabular}

*Significant at $\mathrm{P}<0.05$., **Highly significant at $\mathrm{P} \leq 0.001$.

\section{Discussion}

The congenital missing teeth occur due to disturbance during the initial stage of tooth formation and proliferation. Missing one tooth or more is considered one of the most common developmental anomalies. Moreover, the most common missing type is the bilateral missing lateral incisors ${ }^{(10)}$.

A significant reduction in the arch width between bilateral congenital missing lateral incisors and normal class I occlusion in the maxillary arch was revealed in this study. This could be due to the decrease in the number of the teeth, which in turn causes a reduction in the width of the maxillary $\operatorname{arch}^{(11,12,13)}$.

In the mandibular arch, there was a significant reduction in the intercanine and interpremolar width, but non-significant reduction in the intermolar width was reported the cause of that the missing of the lateral incisors leads to make the canine erupted more mesially than the normal occlusion. This in turn leads to more deficiency of the width of intercanine and interpremolar width $^{(14)}$.

There was a significant reduction in overjet and overbite in bilateral congenital missing lateral incisors compared to normal class I occlusion. This result agrees with that obtained in ${ }^{(15,16)}$, who found that the missing of lateral incisors leads to retroclination of the upper and lower incisor, and thus results in an increase in interincisal angle and more uprighting incisors appearing in the missing lateral incisors patients.

The maxillary arch was significantly greater in males than females in the two groups. This finding is also in agreement with ${ }^{(14,15,17,18,19)}$. This could be due to smaller and smoother bony ridge, the alveolar process of female, or due to weakness of musculature in female ${ }^{(20)}$. This result is not in line with the results obtained by ${ }^{(21,22)}$, who found non-significant differences between males and females. This inconsistency could be due to using different landmarks, different sample size, age group, ethnic group and procedures.

The majority of the dental arch widths in mandibular arch revealed non-significant difference between males and females. This agrees with ${ }^{(23,24)}$, but disagrees with $^{(18,19,20)}$. The difference might be due to racial factor or the difference in the analyzing technique employed.

This study shows non-significant differences based on gender difference in overjet and overbite in the two groups. This result agrees with ${ }^{(24,25,26)}$ : yet, it disagrees with $^{(27,28)}$.

The present study is important to the orthodontist to decide the most appropriate treatment plane to adopt in treating patients with bilateral congenital missing lateral incisors by open or closed the space with maintaining good esthetic to the patient by providing a correct arch width, overjet and overbite. ${ }^{(29)}$ 


\section{Conclusions}

The results indicated a significant reduction in arch width in maxillary and mandibular arch in bilateral missing lateral Incisors when compared with normal class I occlusion group.

There was a significant reduction in overjet and overbite in bilateral missing lateral Incisors as compared with normal class I group.

There was a significant variation between males and females in the two groups in the maxillary arch width. However, non-significant differences were reported in the mandibular arch width.

This study showed non-significant differences based on the gender difference in the overjet and overbite in the two groups.

Ethical Clearance: The Research Ethical Committee at scientific research by ethical approval of both environmental and health and higher education and scientific research ministries in Iraq

Conflict of Interest: The authors declare that they have no conflict of interest.

Funding: Self-funding

\section{Reference}

1. Chu CS, Cheung SL, Smales RJ. Management of congenitally missing maxillary lateral incisors. General Dentistry. 1998 May 1;46(3):268-74.

2. Ramazanzadeh BA, Ahrari F, Hajian S. Evaluation of tooth size in patients with congenitally-missing teeth. Journal of dental research, dental clinics, dental prospects. 2013;7(1):36.

3. Willhite C, Bellerino M, Eubank J. Treatment of congenitally missing lateral incisors with resinbonded fixed partial dentures. Quintessence Dent Technol. 2002;25:63-72.

4. Neville BW, Damm DD, Allen CM and Bouquot JE. Oral \& Maxillofacial Pathology. $3^{\text {rd }}$ ed., Philadelphia: WB Saunder. P. 2009;70-79.

5. Muller TP, Hill IN, Petersen AC, Blayney JR. A survey of congenitally missing permanent teeth. The Journal of the American Dental Association. 1970 Jul 1;81(1):101-7.

6. Graber T.: Orthodontics. Current principle and techniques. 2nd ed. 1994 Mosby St, louis Mosby.
7. Robertsson S, Mohlin B. The congenitally missing upper lateral incisor. A retrospective study of orthodontic space closure versus restorative treatment. The European Journal of Orthodontics. 2000 Dec 1;22(6):697-710.

8. Patel D, Mehta F, Patel N, Mehta N, Trivedi I, Mehta A. Evaluation of arch width among class I normal occlusion, class II division 1, class II division 2, and class III malocclusion in indian population. Contemporary clinical dentistry. 2015 Sep;6(Suppl 1):S202.

9. Mahmood AD. A Comparative study of tooth size and dental arch dimensions between Iraqi Arabs and Kurds with class I normal occlusion. AlRafidain Dental Journal. 2012 Nov 1;12(1):71-9.

10. Proffit WR, Fields HW, Sarver DM. Contemporary orthodontics.(5th edn.) St. Louis: Mosby. 2007.

11. Lavelle CL, Moore WJ. The incidence of agenesis and polygenesis in the primate dentition. American Journal of Physical Anthropology. 1973 May;38(3):671-9.

12. Herzog C, Konstantonis D, Konstantoni N, Eliades T. Arch-width changes in extraction vs nonextraction treatments in matched Class I borderline malocclusions. American Journal of Orthodontics and Dentofacial Orthopedics. 2017 Apr 1;151(4):735-43.

13. Guroo K. Mushtaq M. Comparison of arch width changes in maxilla using two different types of closing loops International Journal of Applied Dental Sciences 2020; 6(3): 77-80.

14. Omar H, Alhajrasi M, Felemban N, Hassan A. Dental arch dimensions, form and tooth size ratio among a Saudi sample. Saudi Medical Journal. 2018 Jan;39(1):86.

15. Øgaard B, Krogstad O. Craniofacial structure and soft tissue profile in patients with severe hypodontia. American Journal of Orthodontics and Dentofacial Orthopedics. 1995 Nov 1;108(5):472-7.

16. Sarnäs KV, Rune B. The facial profile in advanced hypodontia: a mixed longitudinal study of 141 children. The European Journal of Orthodontics. 1983 May 1;5(2):133-43.

17. Hashim HA, Al-Ghamdi S. Tooth width and arch dimensions in normal and malocclusion samples: an odontometric study. J Contemp Dent Pract. 2005 May 15;6(2):36-51.

18. Prasad M, Kannampallil ST, Talapaneni AK, 
George SA, Shetty SK. Evaluation of arch width variations among different skeletal patterns in South Indian population. Journal of natural science, biology, and medicine. 2013 Jan;4(1):94.

19. Golwalkar SA, Msitry KM. An evaluation of dental crowding in relation to the mesiodistal crown widths and arch dimensions. Journal of Indian Orthodontic Society. 2008 Jun; 42(2):22-9.

20. Genecov JS, Sinclair PM, Dechow PC. Development of the nose and soft tissue profile. The Angle Orthodontist. 1990 Sep;60(3):191-8.

21. Raberin M, Laumon B, Martin JL, Brunner F. Dimensions and form of dental arches in subjects with normal occlusions. American journal of orthodontics and dentofacial orthopedics. $1993 \mathrm{Jul}$ 1;104(1):67-72.

22. Lavelle CL, Foster TD. A cross-sectional study into age changes of the human dental arch. Archives of oral biology. 1969 Jan 1;14(1):71-86.

23. Mahmood AD. A Comparative study of tooth size and dental arch dimensions between Iraqi Arabs and Kurds with class I normal occlusion. AlRafidain Dental Journal. 2012 Nov 1;12(1):71-9.
24. Alhuwaizi AF, Al-Mulla AA, A-Alousi, WS. The Overbite of Iraqi Teenagers (A National survey) Iraqi.Orthod J 2005; 1: 1.

25. Cons NC, Mruthyunjaya YC, Pollard ST. Distribution of occlusal traits in a sample of 1337 children aged 15--18 residing in upstate New York. International dental journal. 1978 Jun;28(2):15464.

26. Ciuffolo F, Manzoli L, D'Attilio M, Tecco S, Muratore F, Festa F, Romano F. Prevalence and distribution by gender of occlusal characteristics in a sample of Italian secondary school students: a cross-sectional study. The European Journal of Orthodontics. 2005 Dec 1;27(6):601-6.

27. Agha N, Al-Dawoody A. A Comparative Study of Curve of Spee and Arch Circumference Between Class I Nor-mal Occlusion and Class II Division 1 Ma-locclusion. Al-Rafidain Dental Journal. 2010 Sep 1;10(2):341-7.

28. Cocconi R, Rapa S. Unilateral agenesis of the maxillary lateral incisor: space closure versus space preservation in growing patients. InSeminars in Orthodontics 2020 Feb 21. WB Saunders. 